\title{
Particle Statistics Affects Quantum Decay and Fano Interference
}

\author{
Andrea Crespi, ${ }^{1}$ Linda Sansoni, ${ }^{2, *}$ Giuseppe Della Valle, ${ }^{3,1}$ Alessio Ciamei, ${ }^{2}$ Roberta Ramponi, ${ }^{1,3}$ Fabio Sciarrino, ${ }^{2, \dagger}$ \\ Paolo Mataloni, ${ }^{2}$ Stefano Longhi, ${ }^{3,1, \$}$ and Roberto Osellame ${ }^{1,3,8}$ \\ ${ }^{1}$ Istituto di Fotonica e Nanotecnologie, Consiglio Nazionale delle Ricerche, Piazza Leonardo da Vinci 32, I-20133 Milano, Italy \\ ${ }^{2}$ Dipartimento di Fisica, Sapienza Università di Roma, Piazzale Aldo Moro 5, I-00185 Roma, Italy \\ ${ }^{3}$ Dipartimento di Fisica, Politecnico di Milano, Piazza Leonardo da Vinci 32, I-20133 Milano, Italy
}

(Received 6 October 2014; published 2 March 2015)

\begin{abstract}
Quantum mechanical decay, Fano interference, and bound states with energy in the continuum are ubiquitous phenomena in different areas of physics. Here we experimentally demonstrate that particle statistics strongly affects quantum mechanical decay in a multiparticle system. By considering propagation of two-photon states in engineered photonic lattices, we simulate quantum decay of two noninteracting particles in a multilevel Fano-Anderson model. Remarkably, when the system sustains a bound state in the continuum, fractional decay is observed for bosonic particles, but not for fermionic ones. Complete decay in the fermionic case arises because of the Pauli exclusion principle, which forbids the bound state to be occupied by the two fermions. Our experiment indicates that particle statistics can tune many-body quantum decay from fractional to complete.
\end{abstract}

DOI: 10.1103/PhysRevLett.114.090201

Decay of excited states has been a topic of great interest since the early times of quantum mechanics [1]. A metastable state decaying into a continuum usually shows an exponential decay law and is modeled by a Breit-Wigner resonance, which is the universal hallmark of unstable states. However, more complex manifestations of quantum mechanical decay are observed in the strong coupling regime or when different decay channels are allowed. Strong coupling is responsible for memory effects, leading to deviations from an exponential decay law [2,3], whereas multipath decay channels can lead to Fano interference and asymmetric line shapes (Fano resonances [4,5]). Fano's model $[4,5]$ is a landmark in modern physics. First developed to explain the behavior of electrons scattered by excited atoms [4], it was later adopted to explain phenomena in a number of different physical systems [6], such as ultracold gases and Bose-Einstein condensates (where the Fano resonance is usually referred to as Feshbach resonance [7]), semiconductors, quantum dots and mesoscopic systems [8-10], and plasmonic nanostructures [11]. Fano interference is observed when different decay channels interfere, giving rise to broadening and asymmetric deformations of natural line shapes. In general, the destructive interference between different decay channels is associated with the formation of bound states in the continuum $[4,12]$, which inhibit the complete decay of the excited state. The interplay between bound states in the continuum and Fano-Feshbach resonances has been highlighted in several works (see, for instance, [13-16]). Quantum decay processes [2,3], Fano resonances [4,6], and bound states in the continuum [17-21] have been so far demonstrated in several systems using particles with either bosonic (e.g. neutral atoms, photons) or fermionic
PACS numbers: 42.82.Et, 03.65.Xp, 03.67.Ac, 42.50.-p

(e.g. electrons) nature. However, in such previous experiments the peculiar role of particle statistics in the decay process was not disclosed. Interestingly, recent works [22-28] showed that particle statistics and contact interactions can deeply modify the decay dynamics in a manybody system. Even in the absence of particle interaction, fermions and bosons may show very different decay behavior, in particular in many cases fermions tend to decay faster [22-24]. However, no experimental observation of this phenomenon has been reported yet.

In this work we investigate, experimentally, the decay process of two noninteracting particles to a common continuum, by probing an engineered photonic lattice with two-photon states. The lattice, consisting of a threedimensional array of coupled optical waveguides, is fabricated in a glass substrate by femtosecond laser micromachining [29-31]. While the bosonic dynamics is naturally observed for identically polarized photons, an antisymmetric polarization-entangled state of the two photons is used to simulate the fermionic behavior [32-34], with anyonic statistics in the intermediate regime $[32,33]$. Our experimental results indicate that particle statistics by itself (considering noninteracting particles) is sufficient to tune many-body quantum decay from fractional to complete when the statistics is changed from bosonic to fermionic, respectively.

We focus on systems described by the Fano-Anderson $[4,6]$ or Friedrichs-Lee Hamiltonian [35,36], which is a paradigmatic model to study quantum mechanical decay, Fano interference phenomena, and bound states in the continuum $[12,15,16,27]$. To investigate the role of particle statistics, second quantization of the Fano-Anderson Hamiltonian is required [27]. The simplest case is provided 
by two discrete states coupled to a common tight-binding continuum of modes, i.e. a quantum wire, which are initially occupied by two indistinguishable particles, either bosons or identical fermions [27]. In detail, we consider a system composed of two sites $|1\rangle$ and $|2\rangle$, respectively, with energy $\epsilon_{1}$ and $\epsilon_{2}$, side coupled with hopping rates $\kappa_{1}$ and $\kappa_{2}$, to a common semi-infinite chain of coupled sites (a quantum wire), each with energy $\epsilon=0$ [see Figs. 1(a) and 1(b)]. Thus, states $|1\rangle$ and $|2\rangle$ can decay by tunnelling to the common continuum given by the tight-binding lattice band of the quantum wire. The energy of the band spans the interval $-2 \kappa<E<2 \kappa, \kappa$ being the hopping rate between two adjacent sites of the wire. In the following, $\epsilon_{1}$ and $\epsilon_{2}$ will be considered embedded into the continuum, i.e. $\left|\epsilon_{1,2}\right|<2 \kappa$, and the coupling of the sites $|1\rangle$ and $|2\rangle$ to the continuum will be assumed as weak, i.e. $\kappa_{1,2}<\kappa$.

In our experiment, the sites correspond to optical modes of different waveguides, which interact through their evanescent field. The system is thus formed by two waveguides coupled to a linear waveguide array, according to the geometry of Fig. 1(c). The coupling coefficients $\kappa, \kappa_{1}$, $\kappa_{2}$ depend on the distance between the waveguides and can be tailored by carefully dimensioning the structure. The energies $\epsilon_{1}, \epsilon_{2}$, and $\epsilon$ correspond to the propagation

(a)

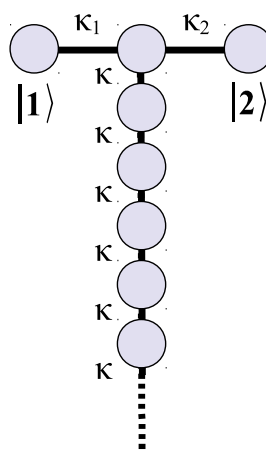

(b)

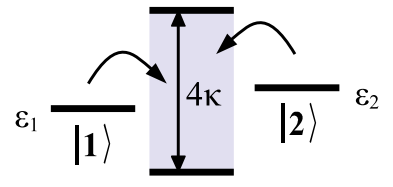

(c)

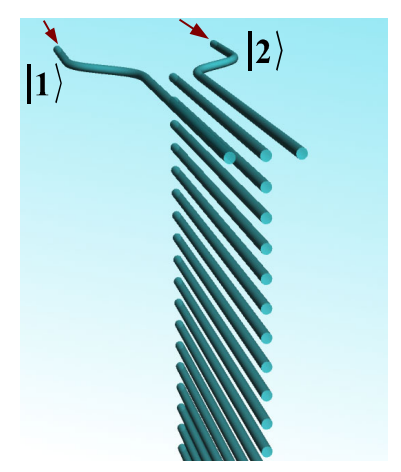

FIG. 1 (color online). Schematic of (a) two quantum wells side coupled to a tight binding quantum wire, and (b) representation of the energy levels. (c) 3D rendering of the actual photonic structure employed in the experiments; two waveguides coupled to a vertical linear array represent the two discrete states coupled to the continuum. The two-photon state is launched in the photonic structure as indicated by the red arrows. constants of the waveguides themselves and can be tuned by varying the refractive index change in the different waveguides (for our direct laser writing process, this is achieved by tailoring the writing speed). In this photonic implementation, the temporal dynamics of the system is mapped onto the propagation distance $z$.

The evolution of the particle-creation operators $a_{j}^{\dagger}$ for the various modes is governed by the coupled-mode equations:

$$
\begin{aligned}
& i \frac{d a_{1,2}^{\dagger}}{d z}=\epsilon_{1,2} a_{1,2}^{\dagger}+\kappa_{1,2} a_{3}^{\dagger}, \\
& i \frac{d a_{3}^{\dagger}}{d z}=\kappa_{1} a_{1}^{\dagger}+\kappa_{2} a_{2}^{\dagger}+\kappa a_{4}^{\dagger}, \\
& i \frac{d a_{j}^{\dagger}}{d z}=\kappa a_{j-1}^{\dagger}+\kappa a_{j+1}^{\dagger}, \quad j \geq 4 .
\end{aligned}
$$

where $a_{1}^{\dagger}$ and $a_{2}^{\dagger}$ refer to the modes $|1\rangle$ and $|2\rangle$, while $a_{j}^{\dagger}$ with $j>2$ refer to the modes of the linear array. The equivalence between the semi-infinite lattice model, described by the operator equations (1), and the FanoAnderson model can be readily established by an operator transformation from the Wannier to the Bloch basis representation [15,16,27]. An interesting property of this system is the existence of one bound state in the continuum when $\epsilon_{1}=\epsilon_{2}$. In fact, it is easy to observe that in this case the operator $b^{\dagger}=e^{i \epsilon_{1} z}\left(a_{1}^{\dagger} / \kappa_{1}-a_{2}^{\dagger} / \kappa_{2}\right)$ satisfies $d b^{\dagger} / d z=0$; i.e. the population of the dressed state described by $b^{\dagger}$ does not decay. In the Bloch basis of operators, the bound state can be interpreted as a result of a destructive Fano interference between different decay channels $[15,16,27]$. This leads to fractional decay when a single particle (photon) is placed in either site (waveguide) $|1\rangle$ or $|2\rangle$. We are studying here the two-particles case, considering an initial state $|\Psi(0)\rangle$ of the system excited with one photon in $|1\rangle$ and one photon in $|2\rangle$. Quantum decay is described by the survival probability:

$$
P_{S}(z)=|\langle\Psi(0) \mid \Psi(z)\rangle|^{2},
$$

which is the probability that, at a propagation distance $z$, both particles are still in the initial state and none has decayed into the continuum. From an experimental point of view, $P_{S}$ corresponds to the probability of coincidence detection of two photons in output modes $|1\rangle$ and $|2\rangle$.

By exciting the system with two identically polarized photons, the natural bosonic behavior is observed. On the contrary, if the system is excited with an antisymmetric polarization-entangled two-photon state, $P_{S}(z)$ has the same expression as if we were injecting two identical particles with fermionic statistics; i.e. the operators $a_{j}^{\dagger}$ satisfy fermionic commutation rules (see Refs. [32,33] and the specific discussion in the Supplemental Material [37]). Figures 2(a) and 2(b) show numerical simulations of 
(a)

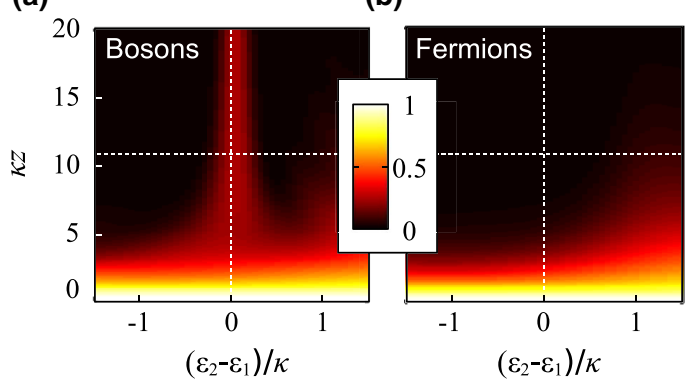

FIG. 2 (color online). Numerical simulations showing the behavior of the survival probability $P_{S}$ for different propagation lengths and energy detuning, normalized with respect to $\kappa$, for the case of (a) bosonic and (b) fermionic particles. Parameter values are $\kappa_{1}=\kappa_{2}=0.37 \kappa$ and $\epsilon_{1}=0.926 \kappa$, which apply to our experiment.

survival probability $P_{S}(z)$ for a system described by (1), in normalized coordinates, for initial two-particle states either bosonic or fermionic. A striking difference between the bosonic and fermionic behavior is evident, as will be experimentally demonstrated in the following.

As a first experiment, we concentrate on the investigation of the quantum decay in the case $\epsilon_{1}=\epsilon_{2}$. We have fabricated several structures as that reported in Fig. 1(c), yielding $\kappa_{1}=\kappa_{2}=0.2 \mathrm{~mm}^{-1}, \kappa=0.54 \mathrm{~mm}^{-1}$, $\epsilon_{1}=\epsilon_{2}=0.5 \mathrm{~mm}^{-1}$, and different lengths $z$ of the array. In our realization the linear array is composed of 25 waveguides. Details on the fabrication process by femtosecond laser direct writing are given in the Supplemental Material [37]. Each structure allows us to photograph the evolution at a specific propagation distance $z$.

The system is first characterized by launching laser light separately in waveguides $|1\rangle$ and $|2\rangle$, and imaging the output facet with a digital camera. The fraction of light remaining in the launch waveguides is measured. This corresponds to investigating the single-particle behavior, when the particle is initially on mode $|1\rangle$ or $|2\rangle$. Survival probability $P_{S \text {,clas }}$ for two classical, distinguishable particles is easily calculated from the product of two singleparticle experimental distributions (since the two particles are uncorrelated).

To experimentally characterize the system behavior for two correlated particles, two photons at $810 \mathrm{~nm}$ wavelength, generated by a spontaneous parametric downconversion source, are coupled to single-mode optical fibers and injected simultaneously in waveguides $|1\rangle$ and $|2\rangle$. Output light from the same waveguides is collected by an objective, coupled to multimode fibers and detected by single-photon avalanche photodiodes [see Fig. 3(a)]. Coincidence-detection counts, in equal temporal gates, are performed for different input states: indistinguishable vertically polarized photons, polarization-entangled photons in an antisymmetric state, and distinguishable photons (the latter being generated by introducing, for each of the previous states, a temporal delay for one of the photons). These conditions correspond to identical bosons, identical fermions, and distinguishable particles. The survival probability for identical bosons $P_{S \text {,bos }}$ and fermions $P_{S \text {,fer }}$ is then retrieved from experimental measurements as follows:

$$
P_{S, \text { bos }}=\frac{C_{V V}}{C_{V V, \text { dist }}} P_{S, \text { clas }}, \quad P_{S, \text { fer }}=\frac{C_{\text {ent }}}{C_{V H, \text { dist }}} P_{S, \text { clas }},
$$

where $C_{V V}$ are the coincidence counts for the $|V V\rangle$ state, $C_{V V \text {,dist }}$ the corresponding counts when one photon is delayed, $C_{\text {ent }}$ are the coincidence counts for the entangled $(|H V\rangle-|V H\rangle) / \sqrt{2}$ state, and $C_{V H \text {,dist }}$ the corresponding counts when one photon is delayed $(\mathrm{H}$ and $\mathrm{V}$ refer to horizontal and vertical polarization, respectively).

Figure 3(b) reports the experimentally characterized survival probabilities for the different $z$ and different input states, compared to numerical simulations for the same system. While $P_{S \text {,bos }}$ shows a fractional decay owing to the existence of a bound state, $P_{S \text {.fer }}$ shows a full decay. The experimental points for fermions do not reach exactly zero due to imperfections in preparing the entangled state and slight polarization dependence of the photonic device. Imperfections in the entangled state preparation could be both in the $\pi$ phase, giving the minus sign in the antisymmetric state, and in a partial photon distinguishability. Respectively, these features induce a partial bosonic (precisely anyonic [32]) or partial classical behavior, both of which introduce a nonvanishing survival probability of the initial state. However, such results clearly show that fractional decay is suppressed for two fermions. This is a signature of the Pauli exclusion principle and can be explained by observing that no more than one fermion can be accommodated into the dressed bound state, while the other fermion necessarily decays into the state continuum.

To better highlight the difference between bosonic and fermionic behavior in the decay process, Fano-like profiles are measured from the survival probability as the detuning of the energy levels of the two discrete states is varied [15,16] (see the Supplemental Material [37] for more technical details). We fabricated and characterized other photonic structures, with fixed length $z=20 \mathrm{~mm}$, the same $\kappa_{1}, \kappa_{2}, \kappa$ as in the previous experiments, $\epsilon_{1}=0.5 \mathrm{~mm}^{-1}$ and different values for $\epsilon_{2}$. Results are shown in Fig. 3(c). For bosonic particles, the survival probability shows a resonance behavior peaked at $\epsilon_{1}=\epsilon_{2}$. This peak is associated with fractional decay observed in the previous experiment and corresponds to perfect destructive interference of the decay channels into the common continuum. At $\epsilon_{2}-\epsilon_{1} \simeq 0.255 \mathrm{~mm}^{-1}$, the survival probability shows a minimum, corresponding to a maximally constructive interference of the decay channels and the largest decay rate (see the Supplemental Material [37]). A similar behavior is found for distinguishable particles. Interestingly 

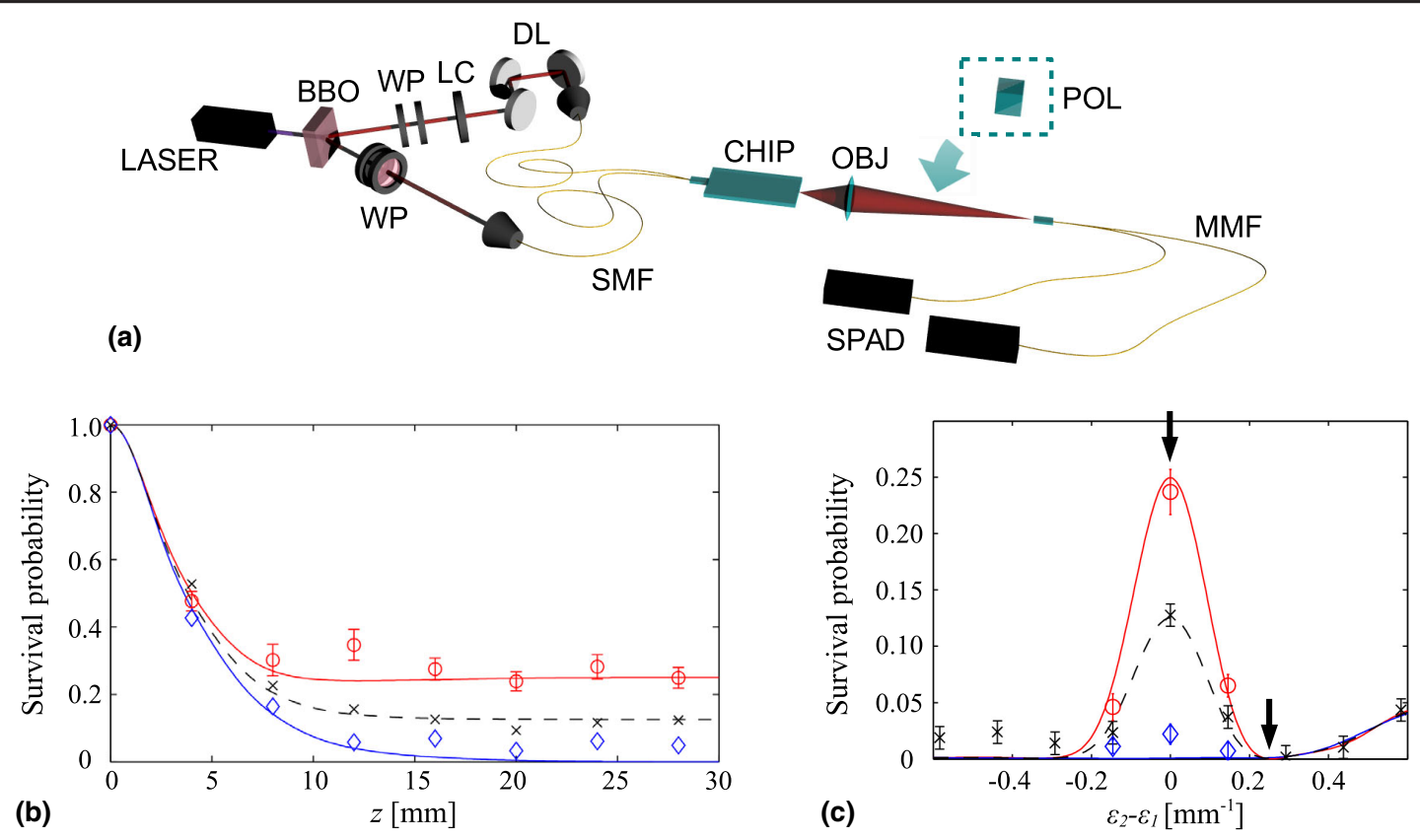

FIG. 3 (color online). (a) Experimental setup for two-photon measurements: two-photon entangled states are generated by spontaneous-parametric down-conversion in a beta-barium borate (BBO) crystal. The generated state is tuned by wave plates (WP) and a liquid crystal retarder (LC); photons are coupled to single-mode fibers (SMF) and injected into the chip. A microscope $5 \times$ objective $(\mathrm{OBJ})$ collects the output light and images the two lateral modes directly onto the entrance facets of two multimode fibers $(\mathrm{MMF})$ in a $250 \mu \mathrm{m}$ pitched fiber array. The fibers are connected to single-photon avalanche photodiodes (SPAD) for detection. A delay line (DL) controls the temporal indistinguishability of the photons. Measurements for $|V V\rangle$ state are performed by injecting a $\frac{1}{\sqrt{2}}(|H H\rangle+|V V\rangle)$ state in the chip and postselecting with a polarizer (POL). (b),(c) Experimental maps of the survival probability for two bosons (red circles), two fermions (blue diamonds), two distinguishable particles (black crosses). Curves of numerical simulation are also shown. In (b) $\epsilon_{2}=\epsilon_{1}=0.5 \mathrm{~mm}^{-1}$ and $z$ is varied, which corresponds to the vertical dashed lines in the maps of Fig. 2. In (c) $z=20 \mathrm{~mm}$ is kept fixed while $\epsilon_{2}$ is varied, which corresponds to the horizontal dashed lines in the maps of Fig. 2. Where not shown, error bars are smaller than marker size. The two arrows in (c) correspond to the energy detunings of destructive $\left(\epsilon_{2}=\epsilon_{1}\right)$ and maximally constructive interference $\left(\epsilon_{2}-\epsilon_{1} \simeq 0.255 \mathrm{~mm}^{-1}\right)$ of the decay channels.

the resonance peak is absent when the two particles possess fermionic statistics, since in this case Pauli exclusion principle forbids the two fermions to be accommodated in the bound state. Such a result is a rather general one and holds whenever the number of bound states in the continuum of the Fano-Anderson Hamiltonian is lower than the number of particles [27].

In conclusion, this work has presented an experimental study on the quantum decay process of two identical particles, initially on discrete states, into a common continuum. A profound difference in the bosonic and fermionic evolution is evidenced; in particular, we found that quantum decay can be tuned from fractional to complete by changing the particle statistics from bosonic to fermionic. This system can be seen as a particle statistics filter, in fact, considering the two initial particles of any statistics, either bosonic or fermionic, after a sufficiently long time only the particles of bosonic statistics will survive in the initial state. The capability to simulate multiparticle dynamics in discrete systems coupled to a continuum may enable the investigation of other multiparticle decay phenomena, such as multiparticle Zeno effects [39] and
non-Markovianity [40]. In addition, the current approach is not only limited to two particles; in fact, proper multiphoton entangled states could be used to reproduce the dynamics of an arbitrary number of bosons or fermions [33].

This work was supported by ERC (European Research Council) Starting Grant 3D-QUEST (3D-Quantum Integrated Optical Simulation, Grant Agreement No. 307783; www.3dquest.eu) and by the European Union through the project FP7-ICT-2011-9-600838 (QWAD Quantum Waveguides Application and Development; www.qwad-project.eu). Partial support from the Fondazione Cariplo is also acknowledged by G. D. V. and S. L. through the project New Frontiers in Plasmonic Nanosensing (Grant No. 2011-0338).

*Current affiliation: Integrated Quantum Optics, University of Paderborn, Warburger Strasse 100, 33098 Paderborn, Germany

†fabio.sciarrino@uniroma1.it 
"stefano.longhi@polimi.it

\$roberto.osellame@ifn.cnr.it

[1] L. Fonda, G. C. Ghirardi, and A. Rimini, Rep. Prog. Phys. 41, 587 (1978).

[2] S. R. Wilkinson, C. F. Bharucha, M. C. Fischer, K. W. Madison, P. R. Morrow, Q. Niu, B. Sundaram, and M. G. Raizen, Nature (London) 387, 575 (1997).

[3] M. C. Fischer, B. Gutierrez-Medina, and M. G. Raizen, Phys. Rev. Lett. 87, 040402 (2001).

[4] U.Fano, Phys. Rev. 124, 1866 (1961).

[5] U. Fano and A. R. P. Rau, Atomic Collisions and Spectra (Academic Press, Orlando, 1986).

[6] A. E. Miroshnichenko, S. Flach, and Y.S. Kivshar, Rev. Mod. Phys. 82, 2257 (2010).

[7] C. Chin, R. Grimm, P. Julienne, and E. Tiesinga, Rev. Mod. Phys. 82, 1225 (2010).

[8] J. Kim, J.-R. Kim, Jeong-O Lee, J. W. Park, H. M. So, N. Kim, K. Kang, K.-H. Yoo, and J.-J. Kim, Phys. Rev. Lett. 90, 166403 (2003)

[9] M. Kroner et al., Nature (London) 451, 311 (2008).

[10] Y. Yoon, M.-G. Kang, T. Morimoto, M. Kida, N. Aoki, J. L. Reno, Y. Ochiai, L. Mourokh, J. Fransson, and J. P. Bird, Phys. Rev. X 2, 021003 (2012).

[11] B. Luk'yanchuk, N. I. Zheludev, S. A. Maier, N. J. Halas, P. Nordlander, H. Giessen, and C. T. Chong, Nat. Mater. 9, 707 (2010).

[12] G. Sudarshan, in Field Theory, Quantization and Statistical Physics edited by E. Tirapegui (D. Reidel Publishing, Dordrecht, Holland, 1988), pp. 237-245.

[13] H. Friedrich and D. Wintgen, Phys. Rev. A 32, 3231 (1985).

[14] P. L. Knight, M. A. Lauder, and B. J. Dalton, Phys. Rep. 190, 1 (1990).

[15] S. Longhi, J. Mod. Opt. 56, 729 (2009)

[16] I. Bayal, B. K. Dutta, P. Panchadhyayee, and P. K. Mahapatra, J. Opt. Soc. Am. B 30, 3202 (2013).

[17] F. Capasso, C. Sirtori, J. Faist, D. L. Sivco, S.-N. G. Chu, and A. Y. Cho, Nature (London) 358, 565 (1992)

[18] P. S. Deo and A. M. Jayannavar, Phys. Rev. B 50, 11629 (1994).

[19] Y. Plotnik, O. Peleg, F. Dreisow, M. Heinrich, S. Nolte, A. Szameit, and M. Segev, Phys. Rev. Lett. 107, 183901 (2011).
[20] S. Weimann, Y. Xu, R. Keil, A. E. Miroshnichenko, A. Tünnermann, S. Nolte, A. A. Sukhorukov, A. Szameit, and Y. S. Kivshar, Phys. Rev. Lett. 111, 240403 (2013).

[21] G. Corrielli, G. Della Valle, A. Crespi, R. Osellame, and S. Longhi, Phys. Rev. Lett. 111, 220403 (2013).

[22] T. Taniguchi and S. I. Sawada, Phys. Rev. E 83, 026208 (2011).

[23] A. del Campo, Phys. Rev. A 84, 012113 (2011).

[24] G. Garcia-Calderon and L. G. Mendoza-Luna, Phys. Rev. A 84, 032106 (2011).

[25] M. Rontani, Phys. Rev. Lett. 108, 115302 (2012).

[26] M. Pons, D. Sokolovski, and A. del Campo, Phys. Rev. A 85, 022107 (2012).

[27] S. Longhi and G. Della Valle, Phys. Rev. A 86, 012112 (2012).

[28] G. Zürn, A. N. Wenz, S. Murmann, A. Bergschneider, T. Lompe, and S. Jochim, Phys. Rev. Lett. 111, 175302 (2013).

[29] J. O. Owens et al., New J. Phys. 13, 075003 (2011)

[30] G. Corrielli, A. Crespi, G. Della Valle, S. Longhi, and R. Osellame, Nat. Commun. 4, 1555 (2013).

[31] K. Poulios et al., Phys. Rev. Lett. 112, 143604 (2014)

[32] L. Sansoni, F. Sciarrino, G. Vallone, P. Mataloni, A. Crespi, R. Ramponi, and R. Osellame, Phys. Rev. Lett. 108, 010502 (2012).

[33] J. C. F. Matthews, K. Poulios, J. D. A. Meinecke, A. Politi, A. Peruzzo, N. Ismail, K. Wörhoff, M. G. Thompson, and J. L. O’Brien, Sci. Rep. 3, 1539 (2013).

[34] A. Crespi, R. Osellame, R. Ramponi, V. Giovannetti, R. Fazio, L. Sansoni, F. De Nicola, F. Sciarrino, and P. Mataloni, Nat. Photonics 7, 322 (2013).

[35] K. O. Friedrichs, Commun. Pure Appl. Math. 1, 361 (1948).

[36] T. D. Lee, Phys. Rev. 95, 1329 (1954).

[37] See Supplemental Material at http://link.aps.org/ supplemental/10.1103/PhysRevLett.114.090201, which includes details on waveguide fabrication and theoretical calculations on survival probability, particle statistics, and Fano line shapes. The Supplemental Material includes Ref. [38].

[38] S. Longhi, Phys. Rev. E 74, 026602 (2006).

[39] X.-B. Wang, J. Q. You, and F. Nori, Phys. Rev. A 77, 062339 (2008).

[40] R. Lo Franco, B. Bellomo, S. Maniscalco, and G. Compagno, Int. J. Mod. Phys. B 27, 1345053 (2013). 